\title{
Synchronization in Wireless Sensors Networks Using balanced clusters
}

\author{
Salim EL KHEDIRI ${ }^{1,3}$, Nejah Nasri ${ }^{1}$ \\ ${ }^{1}$ Laboratory LETI, University of \\ Sfax, National School of Engineers \\ of Sfax, Route Soukra Km 3.5 B.P W \\ 1173, TUNISIA \\ Salim.el.khediri@gmail.com \\ nejah.nasri@isecs.rnu.tn
}

\author{
Abdennaceur Kachouri ${ }^{2}$ \\ ${ }^{2}$ University of Gabes, ISSIG Higher \\ Institute of industrial Systems Gabes \\ CP 6011, TUNISIA \\ Abdennaceur.kachouri@enis.rnu.tn
}

\author{
Anne $\mathrm{Wei}^{3}$ \\ ${ }^{3}$ Center for studies in Computer \\ sciences and communication, \\ CNAM, \\ Anne.wei@cnam.fr
}

\begin{abstract}
The advent of smart devices and continuous expansions of smart environments make Wireless sensor networks (WSNs) an important part of our daily lives. The usage of a myriad of devices in self-organizing networks in a various fields such as home monitoring, medical and military etc. requires an efficient delivery of sensed information. For this necessity, a local clock of sensors nodes needs to be synchronized and up-keep timely synchronization between sensors, to ensue seamlessly communication with each other via radio links aimed at sharing and treatment of reliable information. In this paper, we present balanced Timing-sync protocol for sensor networks that aims at providing network-wide time synchronization in a sensor network. Our schemes work in two steps. In the first step, a hierarchical structure is established in the edges of this structure to establish a global time scale through the network. Ultimately all node in the network synchronize their clock to a reference node. We implement our algorithm on NS2. We argue that our algorithm roughly gives better performance as compared to the work in the same line of research like TPSN.
\end{abstract}

Keywords-Sensors networks, time synchronization, clock drift.

\section{I.INTRODUCTION}

In wireless sensor networks, the aim operation is data fusion, whereby data from each sensor is agglomerated to form a single meaningful result [1]. In general, the proposed technique for synchronization in sensor networks requires that all sensor nodes have a common time scale so that the central unit can coordinate and collaborate sensors to accomplish their tasks. However, it is difficult to maintain a common time scale for all sensors, so the IEEE 802.15.4 standard has not defined clearly the synchronization mechanisms.

Wireless sensor networks (WSNs) can be applied to a wide range of applications in domains as diverse as medical, industrial, military, environmental, scientific, and home networks [2], [4]. Since the sensors in a WSN operate independently, their local clocks may not be synchronized with one another. This can cause difficulties when trying to integrate and interpret information sensed at different nodes. For instance, if a moving car is detected at two different times along a road, before we can even tell in what direction the car is going, the detection times have to be compared meaningfully. In addition, we must be able to transform the two time readings into a common frame of reference before estimating the speed of the vehicle. Estimating time differences across nodes accurately is also important in node localization. For example, many localization algorithms use ranging technologies to estimate inter-nodes distances; in these technologies, synchronization is needed for time-offlight measurements that are then transformed into distances by multiplying with the medium propagation speed for the type of signal used such as radio frequency or ultrasonic. There are additional examples where cooperative sensing requires the nodes involved to agree on a common time frame such as configuring a beam-forming array and setting a TDMA (Time Division Multiple Access) radio schedule [6]. These situations mandate the necessity of one common notion of time in WSNs. Therefore, currently there is a huge research interest towards developing efficient clock synchronization protocols to provide a common notion of time.

The remainder of this paper is structured as follows: the previous work related to the subject of clock synchronization is described in section II, we describe the proposed synchronization algorithm followed by simulation results are presented to verify the superiority of our scheme in section III. The conclusion of our paper is given in section VI.

\section{RELATED WORK}

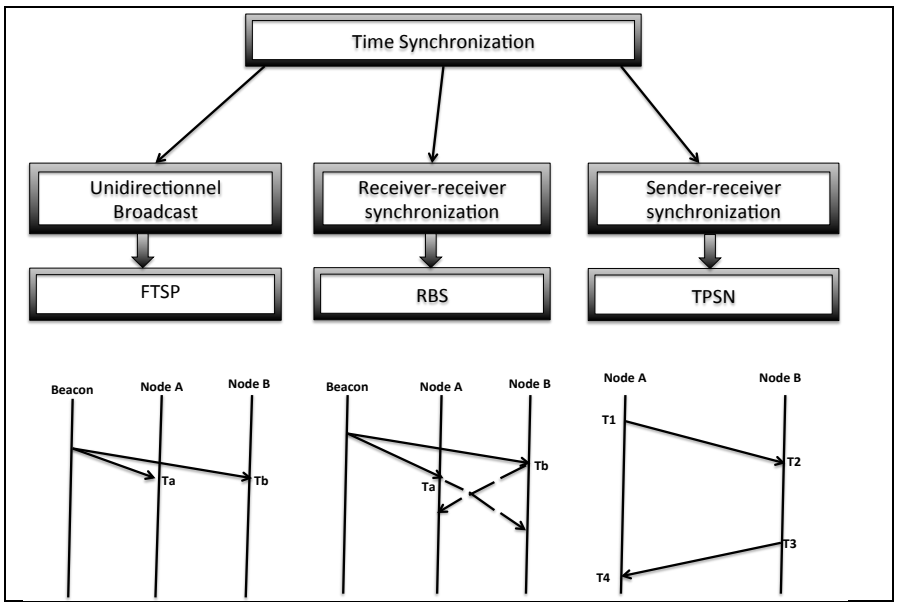

Figure 1. Classification of time synchronization scheme

As illustrated in figure 1 time synchronization algorithm can be roughly classified into three types: (a) simple unidirectional 
broadcast, (b) receiver-receiver synchronization and (c) senderreceiver synchronization.

First in the unidirectional reference broadcast method, with a simple method a reference node simply broadcasts a reference clock signal to other nodes. Which correct their time with the reference clock. This method is the oldest and simplest method for synchronization the network time. The flooding time synchronization protocol (FTSP) [2], [3] is the most known approach that is to achieve a local synchronization with local participating nodes. Assuming that each node has local clock synchronization errors, and can communicate despite the lack of reliability, the errors must be corrected with message exchange mechanism. FTSP synchronize time from a sender to multiple receivers which may be using a single radio message. This mechanism could ensure high accuracy between two sensors and keep synchronized communication. Typically, WSN (Wireless Sensor Network) operates in larger areas than the radius of a node. Therefore, the FTSP synchronizes multihop nodes. The root node is the only selected and dynamic node that maintains the overall time for all other nodes to synchronize their clocks. The nodes form a Ad-hoc structure to transfer the total time from the root to all nodes that keep (save) the initial phase of the tree that is more robust against failures of links between nodes and the dynamic topology change.

Second, receiver-receiver synchronization, Elson and al [5] have proposed the RBS approach which uses the concept of receiver-receiver synchronization. The later has been, viewed as reference to several works in the same line of research (Synchronization Solution). The RBS synchronization mechanism is based on the exploitation of the nature of diffusion of wireless medium. With this property, the nodes in the transmission range of the same location in the intersection of two neighborhoods would be synchronized. Despite the advantages of elimination of major sources of indeterminism, transmitter receive the same message with a very low offset. Considering only the time for receipt of different receptors, the RBS protocol immediately eliminates two major sources of indeterminism involved in the transmission of messages, errors and the precision of synchronization that follows. The mechanism RBS has certain limitations: it requires that the reference receivers of messages transmitted by the reference to know the real time and the advanced channel time. The only source of indeterminism that interfere in RBS synchronization are the propagation and receipt time which shall exchange times of receptions.

Third, sender-receiver, uses the round trip time of the message to correct the offset and propagation delay. In figure 2 we detail an example of the basic operation, which includes three sequential phases. First, node A send it's local time at $t 1$, and node $\mathrm{B}$ receives the message at time $t 2$ and records it's clock, then, time $t 2$ is calculated as $t 2=T 1+d+\delta$, where $d$ the propagation delay between two nodes, and $\delta$ is a clock offset denote between them. Finally, node A can calculate the clock offset and propagation delay between two nodes as below:

\begin{tabular}{l}
$\mathrm{D}=(\mathrm{T} 2-\mathrm{T} 1)+(\mathrm{T} 4-\mathrm{T} 3) / 2$ \\
$\delta=(\mathrm{T} 2-\mathrm{T} 1)-(\mathrm{T} 4-\mathrm{T} 3) / 2$ \\
\hline
\end{tabular}

Figure 2. Offset and propagation delay

Several studies using this mechanism, include for example: Timing-sync Protocol for Sensor Network (TPSN) [2], [4], Arnewel and al have proposed an alternative approach to synchronization with the type Transmitter-receiver, TPSN is a hierarchical algorithm which works on two different phases: The discovery and synchronization phase. In the first phase, we give a network node level. The node that initiates the synchronization is called the root node with the value of level zero neighbors with $n$ hops $(n=1,2,3, K)$ have the value of level $\mathrm{n}$. This process continues until all neighbors attribute their levels. In second phase, a pair wise synchronization is performed along the edges of the hierarchical structure up to a total synchronization of the tree constructed with the message exchange mechanism.

\section{PROPOSED APPROACH}

\section{A. PROPOSED APPROACH}

Our approach is based on the ideas mentioned in [3], [4], when number of levels increases, local clock offset difference also increase. For this reasons our scheme based on balanced clustering into network to minimize complexity, and

As shown in figure 3, the network was comprised of a root node, cluster head nodes and many simple nodes (non-clusterhead members).

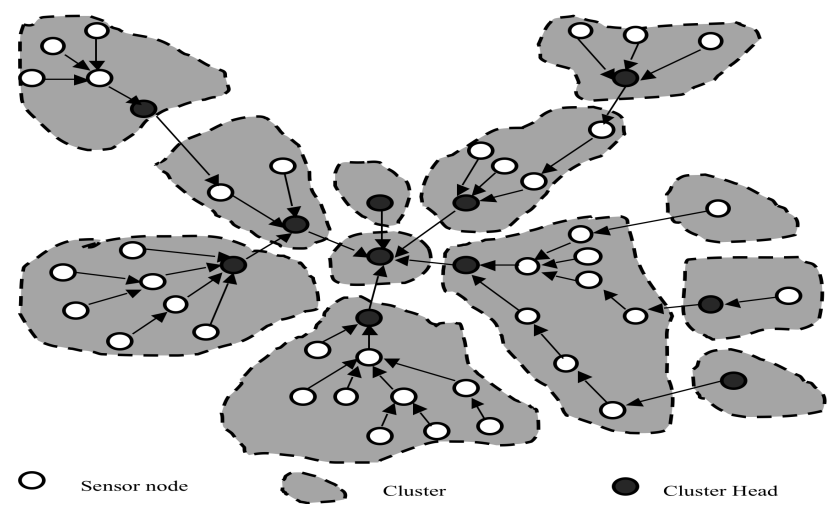

Figure 3. Tree divided in balanced clustering

Some of the assumptions made in clustered time synchronization in wireless sensor network are as following:

- The network composed by $\mathrm{N}$ sensor nodes deployed in square field and has formed cluster hierarchical topology.

- The root node is located inside the sensing field and their level predetermined at level-0.

- Nodes are location-unaware.

- The cluster head nodes are aware of its members and can directly communicate with them. 
- The cluster head are aware of their parent cluster heads

- Each node is synchronized with its $\mathrm{CH}$.

Figure 4 describe the algorithm of the proposed synchronization approach:

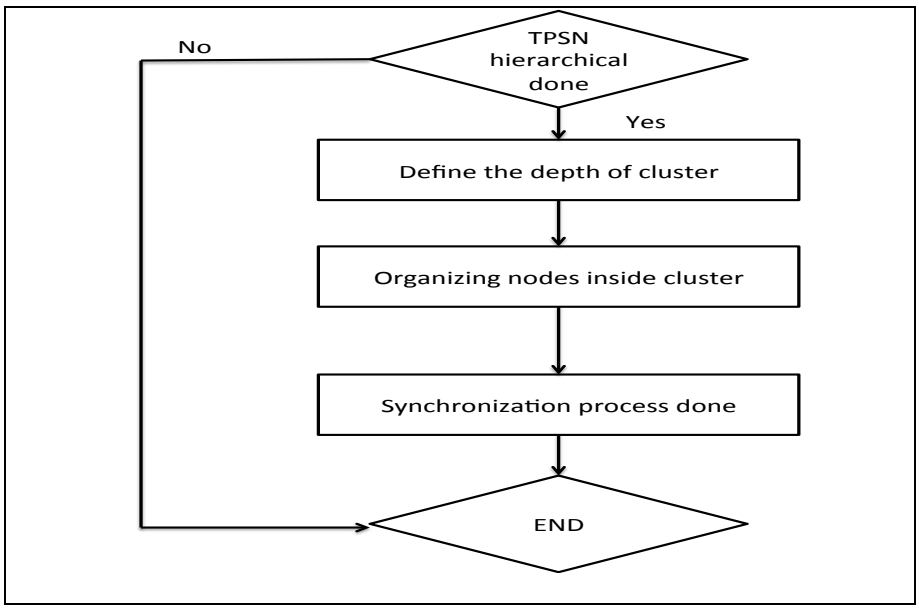

Figure 4. Algorithm Diagram

First, we define the same tree as TPSN [4], after our improvement is to divide the tree into cluster. After this step we can find unbalanced clusters for this reasons a level depth well be used. In fig 5 we can show a simple example of the network after the process of organization is done. In this paper we use 4 as logical level depth to improve the efficacy of our idea, and other depth value can also be used. all depends on the size of the tree.

Our goal is to synchronize the clocks of all the child nodes with the clock of the root node.

After forming the hierarchical network for WSN, synchronization is achieved through bridge nodes, which supports parameters of sources nodes to destination, and update all the nodes on the path. We can explain the scenario by a simple example. For example node $\mathrm{A}$ is the requester synchronization, node $\mathrm{B}$ the bridge and $\mathrm{C}$ is the root to be synchronized. When node A start synchronization, it asks for the time to its immediate ascendant which is the node $\mathrm{B}$, node $\mathrm{B}$ answers this request packet with acknowledge packet, however using the bridge synchronization request to node $\mathrm{C}$. When node $\mathrm{C}$ receives the forward packet from $\mathrm{B}$, the node $\mathrm{C}$ request with it is time information. When node $\mathrm{B}$ receive the packet. Update its clock, after this phase $\mathrm{B}$ replace the requester sent time with requester stored from $\mathrm{A}$, and forwards acknowledge packet to node A, with this packet node A update its clock correctly.

We can summarize our approach into 2 major steps:

1. Level Discovery phase

a. Create a TPSN hierarchy

b. Define the number of children for each parent. c. Define the depth for each cluster

2. Synchronization phase

a. Synchronization inter cluster

b. Global synchronization

\section{B. SIMULATION AND ANALYSES}

This section compares the performance of proposed algorithm with TPSN synchronization protocol. The performance evaluation includes three parts: Clock offset, received/sent message. The sensors are simulated to deploy over the square sized area of $100 \mathrm{~m} \times 100 \mathrm{~m}$.

Simulation is performed using NS2.34, a discrete event network simulator. We have compared the performance of our scheme with TPSN because there are in the same axe of research, based on above energy analysis. The basic parameters used are listed in Table-1.

TABLE 1: SIMULATION PARAMETERS

\begin{tabular}{|l|l|}
\hline Routing Protocol & DSDV \\
\hline Speed of movement of nodes & $1 \mathrm{~m} / \mathrm{s}$ \\
\hline Pause time of nodes & $20 \mathrm{~s}$ \\
\hline Type of antenna & Omni Antenna \\
\hline Type of canal & Wireless Channel \\
\hline Model of the radio propagation & TwoRayGround \\
\hline Model of MAC layer & Mac/802_11 \\
\hline
\end{tabular}

Figure 5 shows the clock difference on each node in wireless sensors networks.

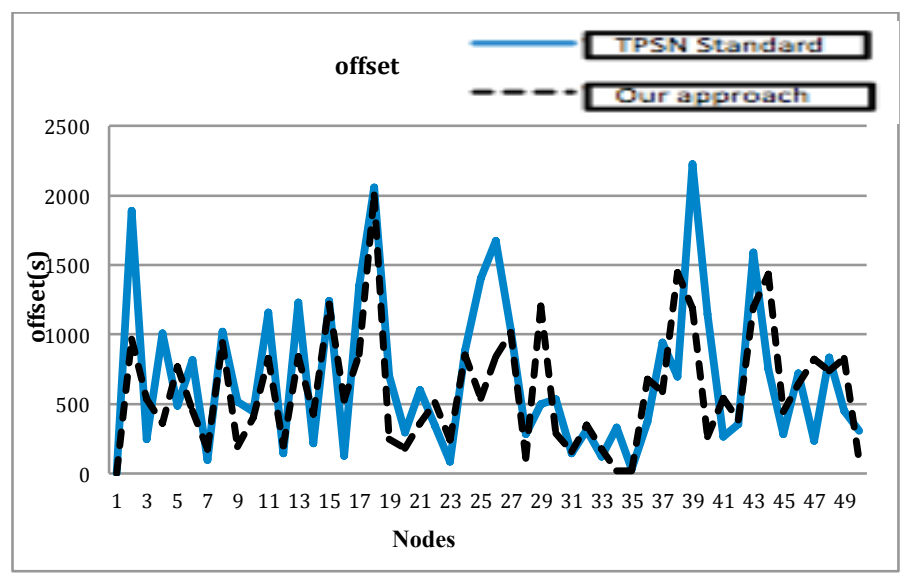

Figure.5. Offset comparison between TPSN standard and improved TPSN

As TPSN causes bigger clock difference at higher levels, but after dividing the network into clusters and made synchronization between cluster roots the clock offset is decreased and the precision increased. 


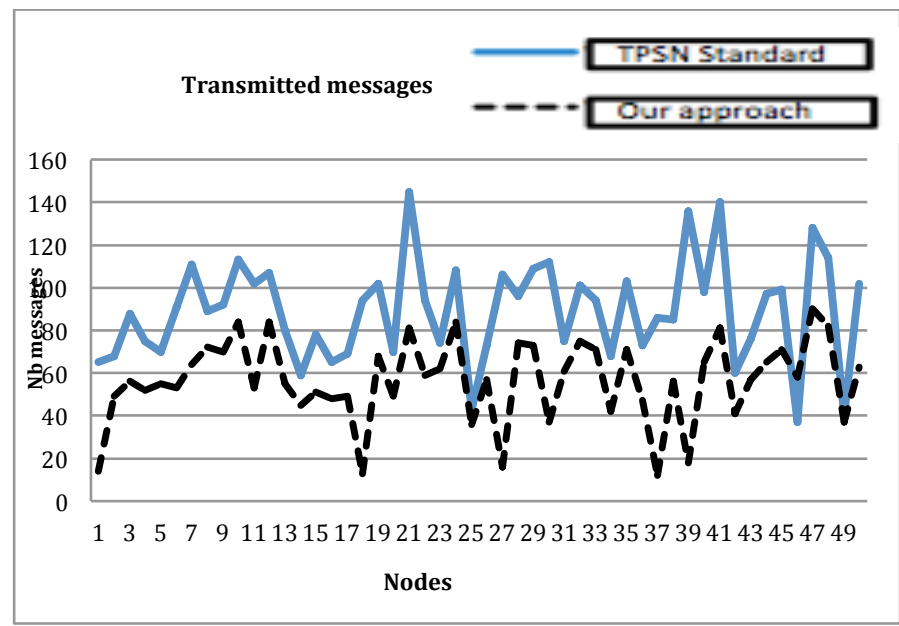

Figure.6. Transmitted messages

Figure.6.shows the numbers of message exchange between our scheme and TPSN for 50 nodes, it's clear that the difference was due to equilibrate clustering of nodes into the network. So, due to clustering the sensor nodes within the cluster message exchange is also less as compare to the TPSN. Table. 2 resumes the performance of our scheme.

\section{TABLE.2: COMPARISON BETWEEN OUR IMPROVEMENT AND TPSN}

\begin{tabular}{|c|c|}
\hline TPSN & PROPOSED APPROACH \\
\hline It is a distributed clustering algorithm & $\begin{array}{l}\text { It is a selective clustering } \\
\text { algorithm }\end{array}$ \\
\hline $\begin{array}{l}\text { A simple and effective way of time } \\
\text { synchronization in the WSN }\end{array}$ & $\begin{array}{l}\text { A good precision improvement } \\
\text { with low message overheads }\end{array}$ \\
\hline $\begin{array}{l}\text { No balancing number of nodes into } \\
\text { cluster }\end{array}$ & $\begin{array}{c}\text { Balancing number of nodes into } \\
\text { cluster }\end{array}$ \\
\hline $\begin{array}{l}\text { It is neither guaranteed that desired } \\
\text { number of cluster heads will be } \\
\text { formed nor even distribution of } \\
\text { cluster heads in the network }\end{array}$ & $\begin{array}{l}\text { It is guaranteed that desired } \\
\text { number of cluster heads will be } \\
\text { able to accomplish their tasks } \\
\text { into network }\end{array}$ \\
\hline $\begin{array}{l}\text { Life time of network will be less } \\
\text { compared to that of BTS }\end{array}$ & $\begin{array}{l}\text { Life time of the network will be } \\
\text { more compared to that of TPSN }\end{array}$ \\
\hline $\begin{array}{l}\text { Start up energy dissipation will be } \\
\text { less compared to that of BTS }\end{array}$ & $\begin{array}{l}\text { Start up energy dissipation will } \\
\text { be more compared to that of } \\
\text { TPSN }\end{array}$ \\
\hline $\begin{array}{l}\text { no tidying up method to remove extra } \\
\text { message overhead }\end{array}$ & $\begin{array}{l}\text { Tidying up method to remove } \\
\text { extra message overhead }\end{array}$ \\
\hline
\end{tabular}

\section{CONCLUSION AND FURTHER IMPROVEMENTS}

Time synchronization is an essential part of infrastructure in sensor network. In this article, we have introduced an algorithm based on the method of clustering for network-wide time synchronization in wireless sensor network. Our scheme is based on the sender-receiver time synchronization approach. We discoursed that unlike traditional wireless network, the sensor network approach of sender-receiver synchronization is better than receiver-receiver synchronization approach. We supported our assertion by comparing our proposition with TPSN, and the superior performance achieved compared to TPSN. Since previous research [5], proved that TPSN gives good precision than Reference broadcast synchronization (RBS), we have verified the effectiveness of this analysis by implementing our idea and TPSN on NS2.34.

We plan to perform further experiments in networks with a magnitude larger number of nodes (thousands), and also to conduct test with mobile node.

\section{REFERENCES}

[1] EL. Salim, N. NASRI, Anne WEI and A. KACHOURI, "Survey of Synchronization Algorithms: Wireless Sensor Networks Case Study," The second international conference on communication and information Technology (ICCIT 2012). Tunisia, June 2012.

[2] EL. Salim, N. NASRI and A. KACHOURI, "Diverses Synchronization Issues in Wireless Sensor Networks," The 23rd international conference on microelectronics (ICM 2011). Tunisia, December 2011.

[3] M.Maroti, B.Kusy, G.Simon and A.Ledeczi, The Flooding Time Synchronization Protocol, Proceedings. of the 2nd ACN Conference on Embedded Networked Sensor Systems (SenSys), Baltimore, Maryland, pp. 3949. 2004.

[4] S.Ganeriwal, R.Kumar and Srivastava, M. B, "Timing-Sync Protocol for Sensor Networks," The First ACM Conference on Embedded Networked Sensor System (SenSys), p. 138-149, November 2003.

[5] J. E.Elson, L.Girod and D.Estrin, "Fine-Grained Network Time Synchronization using Reference Broadcasts." The Fifth Symposium on Operating Systems Design and Implementation (OSDI), p.147-163, December 2002.

[6] G.C.Gautam, T.P.Sharma, V.Katiyar, and A.Kumar, "time synchronization Protocol for Wireless Sensor Network using Clustering," IEE-International Conference on recent trends in information technology, ICRTIT. China, June 2011.

[7] S.Rahamatkar and Dr. A. Agarwal, "A reference Based, Tree Structured Time Synchronization Approach and Its Analysis In WSN, " International Journal of Ad hoc, Sensor and Ubiquitous Computing (IJASUC) Vol.2, No.1, March 2011

[8] LI.Ming He, 2008, "Time Synchronization Based on Spanning Tree for Wireless Sensor Network, " EEE Wireless 4th International Conference on Communication, Networking and Mobile Computing,WiCOM,08

[9] P. Sommer, R.Wattenhofer,"Gradient Clock Synchronization in Wireless Sensor Networks," Computer Engineering and Networks Laboratory, San Francisco, California, USA, IPSN'09, April 13-16, 2009.

[10] P. Ranganathan, K. Nygard, "Time Synchronization in Wireless Sensor Networks : A Survey," International journal of UbiComp (IJU), 1(2), pp 92-102, 2010. 